\title{
Clinical significance and biological mechanisms of glutathione S-transferase mu gene family in colon adenocarcinoma
}

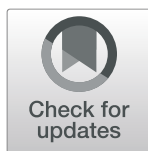

\author{
Erna Guo ${ }^{1,2^{*}}$ D, Haotang $\mathrm{Wei}^{3}$, Xiwen $\mathrm{Liao}^{4}$, Liuyu $\mathrm{Wu}^{1}$ and Xiaoyun Zeng ${ }^{1 *}$
}

\begin{abstract}
Background: Colon adenocarcinoma (COAD) is the most common form of colon cancer. The glutathione Stransferase Mu (GSTM) gene belongs to the GST gene family, which functions in cell metabolism and detoxification. The relationship between GSTM and COAD and the underlying mechanism remain unknown.

Methods: Data extracted from The Cancer Genome Atlas included mRNA expression and clinical information such as gender, age, and tumor stage. Prognostic values of GSTM genes were identified by survival analysis. Function and mechanism of prognostic GSTM genes were identified by gene set enrichment analysis. A nomogram was used to predict the contribution of risk factors to the outcome of COAD patients.

Results: Low expression of GSTM1 and GSTM2 was related to favorable OS (adjusted $P=0.006$, adjusted HR $=0.559$, $95 \% \mathrm{Cl}=0.367-0.849$ and adjusted $P=0.002$, adjusted $\mathrm{HR}=0.519,95 \% \mathrm{Cl}=0.342-0.790$, respectively) after adjusting for tumor stage. Enrichment analysis also showed that genes involved were related to cell cycle, metabolism, and detoxification processes, as well as the Wnt signaling and NF-KB pathways.
\end{abstract}

Conclusions: In conclusion, low expression of GSTM1 and GSTM2 were significantly associated with favorable prognosis in COAD. These two genes may serve as potential biomarkers of COAD prognosis.

Keywords: GSTM, Prognosis, mRNA, Expression, Colon adenocarcinoma

\section{Background}

Colon adenocarcinoma (COAD) is the most common form of colon cancer. There were 140,250 estimated new cases and 50,630 estimated deaths in 2018, and the five years survival rate is $64.5 \%$ as determined by the Surveillance, Epidemiology, and End Results Program (SEER; https://seer.cancer.gov) [1]. Alcohol consumption, smoking, and obesity are risk factors for colorectal cancers [2-4]. Identifying appropriate biomarkers for COAD patients prognosis is important. The glutathione S-transferase $M u(G S T M)$ gene family belongs to the

\footnotetext{
*Correspondence: guoerna@gxmu.edu.cn; zxyxjw@21cn.com

'School of Public Health, Guangxi Medical University, 22 Shuang Yong Road, Nanning 530021, Guangxi Zhuang Autonomous Region, People's Republic of China

Full list of author information is available at the end of the article
}

GST sub-family, which plays important roles in cell metabolism and detoxification [5-7]. GSTM is encoded by five genes (GSTM1-5) [8-11]. However, the correlation of GSTM with the prognosis of cancers is not clear, and there are no reports about the relationship between the GSTM family and COAD. In the present study, we investigated the expression of the GSTM gene family in COAD, and performed a survival analysis including clinical data. A nomogram model was used to predict the outcome of COAD, and joint-effects survival analysis was carried out to show that low expression of GSTM1 and GSTM2 was a sensitive predictor of favorable prognosis. Gene set enrichment analysis (GSEA) and serval enrichment analysis were performed to clarify the

C C The Author(s). 2020 Open Access This article is licensed under a Creative Commons Attribution 4.0 International License, which permits use, sharing, adaptation, distribution and reproduction in any medium or format, as long as you give appropriate credit to the original author(s) and the source, provide a link to the Creative Commons licence, and indicate if changes were made. The images or other third party material in this article are included in the article's Creative Commons licence, unless indicated otherwise in a credit line to the material. If material is not included in the article's Creative Commons licence and your intended use is not permitted by statutory regulation or exceeds the permitted use, you will need to obtain permission directly from the copyright holder. To view a copy of this licence, visit http://creativecommons.org/licenses/by/4.0/ The Creative Commons Public Domain Dedication waiver (http://creativecommons.org/publicdomain/zero/1.0/) applies to the data made available in this article, unless otherwise stated in a credit line to the data. 
potential function and prognostic value of low GSTM1 and GSTM2 expression.

\section{Methods}

\section{Data preparation}

The patient's individual prognosis information was downloaded from University of California, Santa Cruz Xena browser (UCSC Xena: http:// xena.ucsc.edu/, accessed Oct. 5th, 2018). The mRNA expression data for the analysis were generated from The Cancer Genome Atlas (TCGA, http://tcga-data.nci.nih.gov/tcga, accessed Oct. 1th, 2018). Clinical information of 438 patients including gender, age, and tumor stage were selected after deleting cases with missing survival status and survival time of 0 days.

\section{Bioinformatics analysis}

To understand the distribution of GSTM genes between COAD tumor and normal tissues, boxplots were generated from Gene Expression Profiling Interactive Analysis (GEPIA, http://gepia.cancer-pku.cn/, accessed Oct. 2,
2018) [12]. The Database for Annotation, Visualization, and Integrated Discovery (DAVID) v.6.8 (https://david. ncifcrf.gov/tools.jsp, accessed Oct. 11, 2018) $[13,14]$ and BiNGO (https://www.psb.ugent.be/cbd/papers/BiNGO/ Home, accessed Oct.12, 2018) [15] were used to analyze functional enrichment.

\section{Correlation and association analyses}

A gene function prediction website (GeneMANIA: http://genemania.org/, accessed Oct. 15, 2018) was used to analyze interactions among GSTM family members [16]. As well as The Search Tool for the Retrieval of Interacting Genes/Proteins (STRING) database (http:// string.embl.de/; accessed Oct.15, 2018), and those with a required interaction score $>0.15$ were considered statistically significant [17].

\section{Correlation matrix of GSTM genes in COAD}

Pearson's correlation coefficient ( $r$ ) is used to evaluate the association between GSTM genes in COAD. A correlation coefficient $r \geq 0.4$ or $r \leq-0.4$ was considered to
A

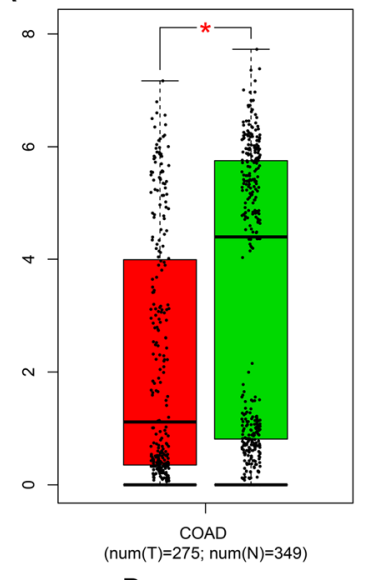

$\mathrm{D}$

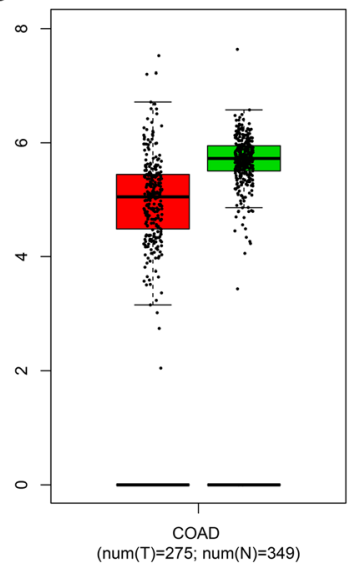

B

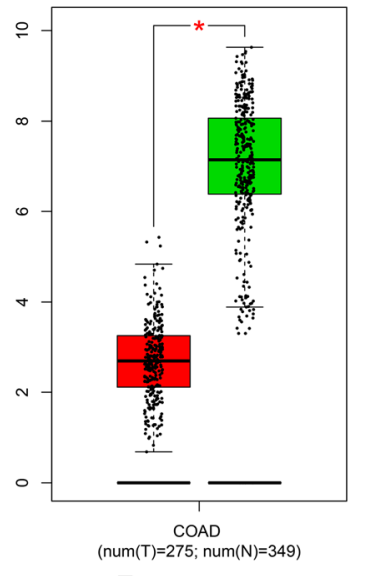

$\mathrm{E}$
C

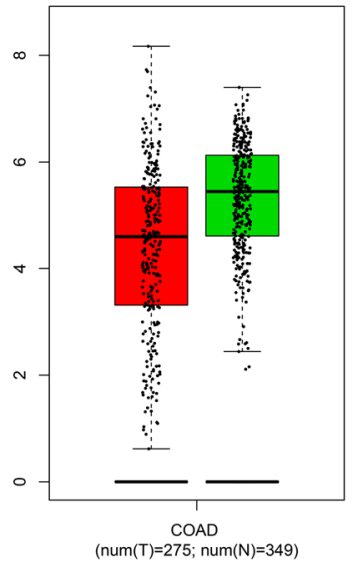

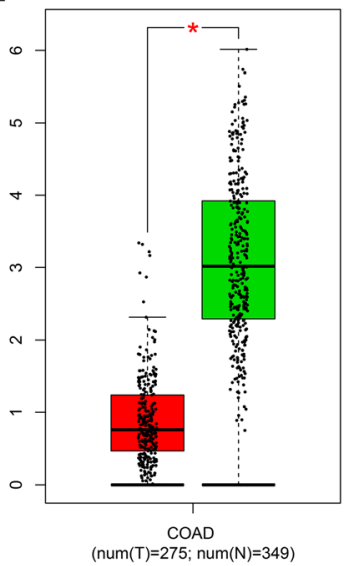

Tumor tissue

Normal colon tissue

Fig. 1 Boxplots for GSTM gene family expression in colon tumor and normal tissues by Gene Expression Profiling Interactive Analysis (GEPIA). (a) GSTM1; (b) GSTM2; (c) GSTM3; (d) GSTM4; (e) GSTM5 
reflect a high correlation. $P$ value less than 0.01 was considered statistically significant.

\section{Clinical significance of GSTM genes in COAD}

For each GSTM gene, patients were evenly divided into high- and low-expression groups by median expression. The Kaplan-Meier estimator was applied to identify correlations between the genes and patient overall survival (OS). Multivariate Cox proportional hazards regression model was adjusted for tumor stage.

\section{Nomogram for predicting the prognosis of COAD}

A nomogram was generated to predict the prognostic outcome and risk rank. All GSTM genes and clinical information were included in the nomogram model. Points were positively correlated with risk, and the points corresponding to each parameter were assessed. Prognosis was predicted at 1,5 and 10 years [18].

\section{Joint-effects survival analysis}

In order to further improve the prognostic ability of GSTM genes in COAD OS, we further analyzed the combined effects of prognostic GSTM genes combinations.

\section{Gene set enrichment analysis (GSEA)}

Biological function differences between GSTM gene phenotypes with different expression levels were explored using GSEA v.3.0 with reference to gene sets of c2 (c2.all.v6.1.symbols.gmt) and c5 (c5.all.v6.1.symbols.gmt) gene set, respectively [19]. Enrichment results meeting $P<0.05$ and a false discovery rate $(\mathrm{FDR})<0.25$ were considered to be significantly different between the two groups.

\section{Statistical analysis}

Hazard ratios (HRs) and 95\% confidence intervals (CIs) were used to assess the risk ratios of survival differences between groups. $P<0.05$ was considered to be

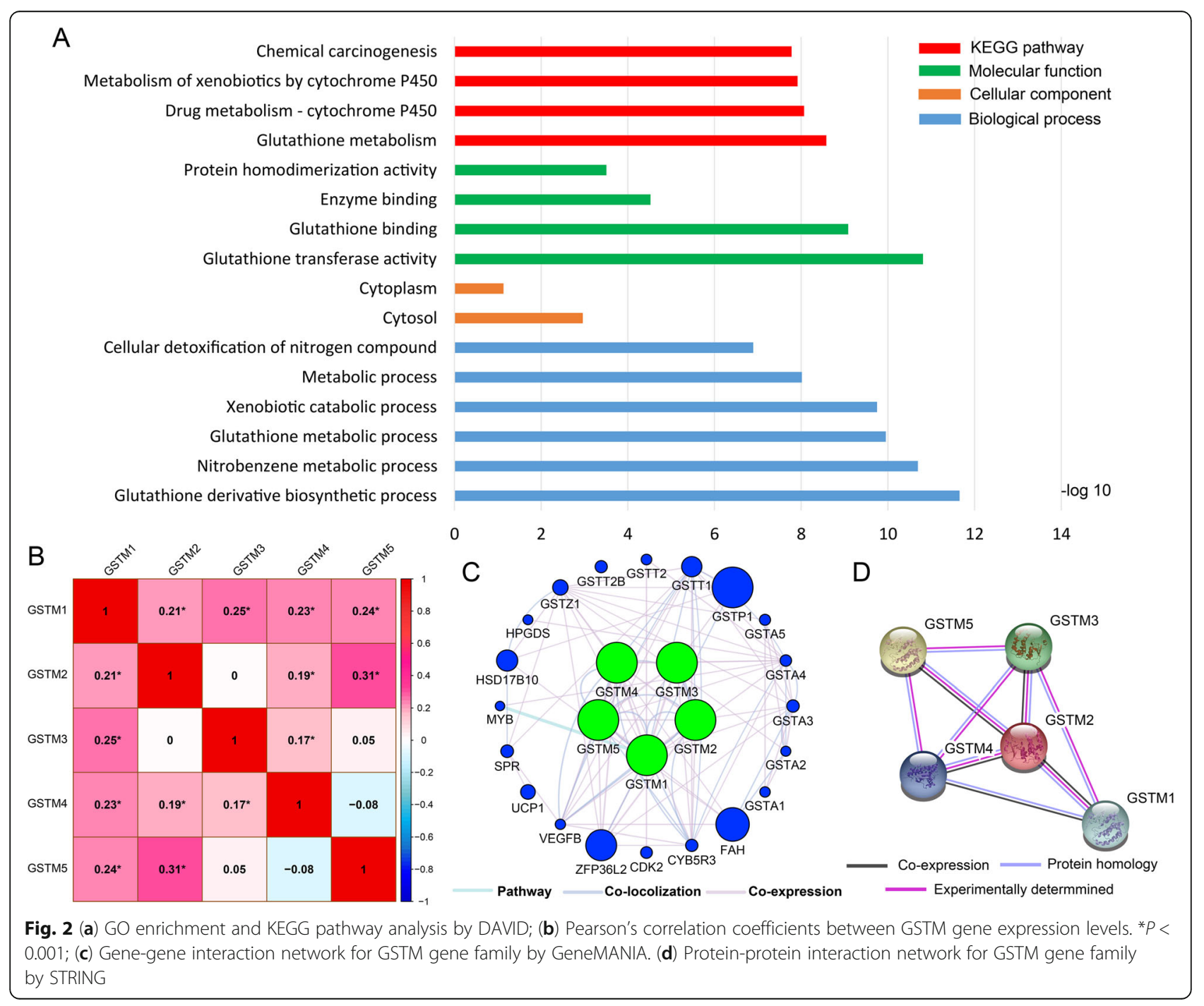


significantly different between groups. SPSS v.25.0 (IBM, Chicago, IL, USA) and GraphPad v.7.0 (La Jolla, CA, USA) are used for statistical analysis and figures drawing respectively. Figures plotting was performed by R v.3.5.1 and Cytoscape v3.6.1 [20].

\section{Results}

Data analysis

After selection, 438 cases were included in the analysis (Table S1). Tumor stage was the only factor associated with favorable prognosis; earlier tumor stages were associated with a more favorable prognosis. Expression profile of GSTM genes are summarized in Fig. 1. GSTM1, GSTM2, and GSTM3 were expressed at significantly higher levels in normal tissues than in colon tumor tissues (Fig. 1A, B, and E).

\section{Functional enrichment analysis of GSTM genes}

Functional enrichment of GSTM genes were evaluated for gene ontology (GO) functional and Kyoto Encyclopedia of Genes and Genomes (KEGG) pathway analyses (Fig. 2A). The results of BiNGO enrichment analysis are shown in Fig. S1. There were no results for $\mathrm{CC}$ in this enrichment. The GSTM family was involved in metabolic processes and glutathione-related processes including metabolism, transfer, and binding. Correlation analysis between the GSTM family is shown in Fig. 2B. There were no significant associations between GSTM2 and GSTM3, GSTM3 and GSTM5, or GSTM4 and GSTM5. The other genes were markedly related to each other $(P<0.01)$. The correlation of GSTM1 and GSTM2 with matrix gene expression is shown in Figs. S2 and S3 (all $P<0.05$ and correlation coefficient $>0.4$ ). Coexpression analysis of the GSTM family at the mRNA level by GeneMANIA is shown in Fig. 2C. The PPI network determined by STRING is shown in Fig. 2D.

\section{Survival analysis}

Vertical scatter plots for the expression of the GSTM genes are shown in Fig. 3. Differences between high- and low-expression groups were markedly difference (all $P<$ 0.05). Survivorship curves of GSTM genes are summarized in Fig. 4A-E. Only low expression of GSTM1 and GSTM2 was markedly related to favorable prognosis $(P=0.018, \mathrm{HR}=0.614,95 \% \mathrm{CI}=0.410-0.919$, Fig. 4A; $P=0.003, \mathrm{HR}=0.545,95 \% \mathrm{CI}=0.364-0.818$, Fig. 4B, respectively). The multivariate Cox proportional hazard regression model only included tumor stage. The results are summarized in Table 1 . The results of univariate survival analysis were consistent with those of multivariate survival analysis: low expression of GSTM1 and GSTM2 was markedly related to favorable OS (adjusted $P=$ 0.006 , adjusted $\mathrm{HR}=0.559,95 \% \mathrm{CI}=0.367-0.849$; adjusted $P=0.002$, adjusted $\mathrm{HR}=0.519,95 \% \mathrm{CI}=0.342$ 0.790 , respectively).

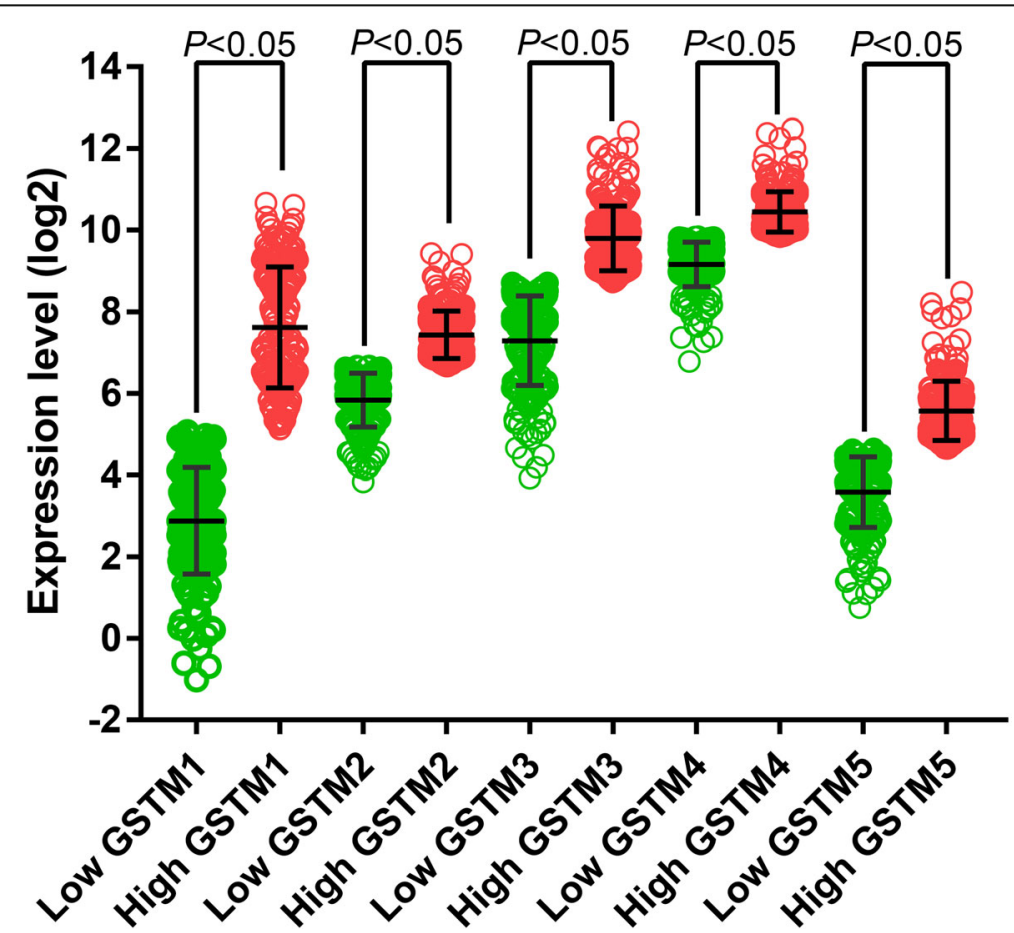

Fig. 3 Scatter plots for GSTM1, GSTM2, GSTM3, GSTM4, and GSTM5 gene expression levels in The Cancer Genome Atlas 

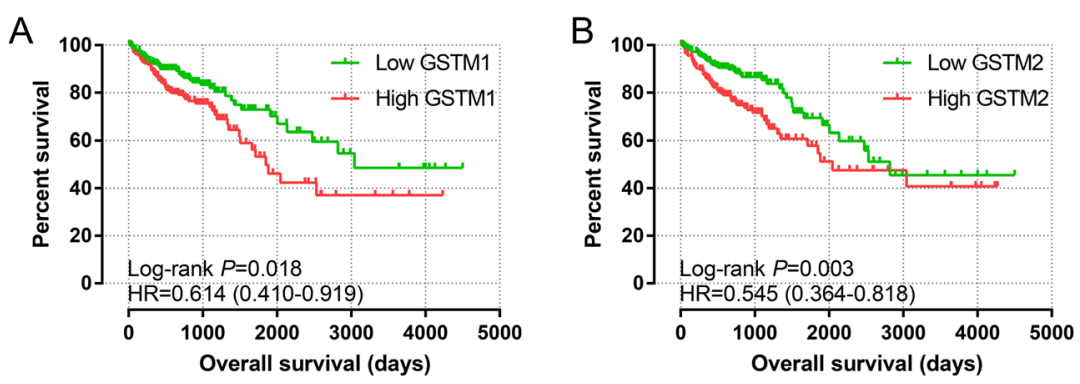

C

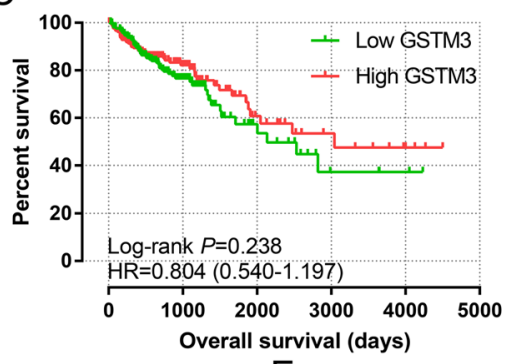

D

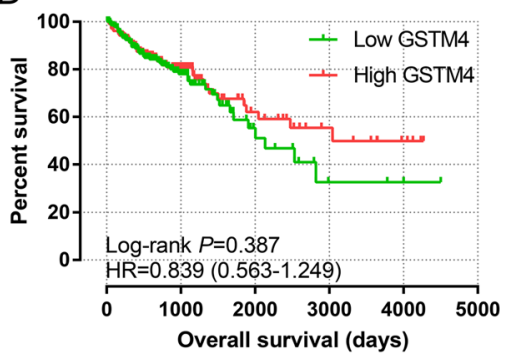

E

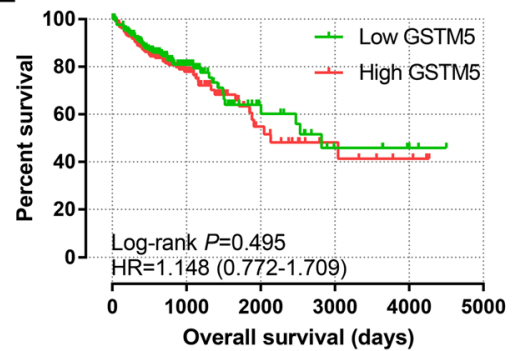

Fig. 4 Prognostic value of GSTM expression for OS. (a-e) Kaplan-Meier survival curves for COAD patients according to GSTM1 (a), GSTM2 (b), GSTM3 (c), GSTM4 (d), and GSTM5 (e) expression $(n=438)$

Table 1 Prognostic survival analysis results

\begin{tabular}{|c|c|c|c|c|c|c|c|}
\hline Gene & $\begin{array}{l}\text { Patients } \\
(n=438)\end{array}$ & No. of events (\%) & $\begin{array}{l}\text { MST } \\
\text { (days) }\end{array}$ & $\begin{array}{l}\text { Crude HR } \\
(95 \% \mathrm{Cl})\end{array}$ & Crude $P$ & $\begin{array}{l}\text { Adjusted HR* } \\
(95 \% \mathrm{Cl})\end{array}$ & Adjusted P* \\
\hline \multicolumn{8}{|l|}{ GSTM1 } \\
\hline High & 219 & $57(26.0 \%)$ & 1849 & Ref. & 0.018 & Ref. & 0.006 \\
\hline Low & 219 & 41 (18.7\%) & 3042 & $0.614(0.410-0.919)$ & & $0.559(0.367-0.849)$ & \\
\hline \multicolumn{8}{|l|}{ GSTM2 } \\
\hline High & 219 & $59(26.9 \%)$ & 2047 & Ref. & 0.003 & Ref. & 0.002 \\
\hline Low & 219 & $39(17.8 \%)$ & 2821 & $0.545(0.364-0.818)$ & & $0.519(0.342-0.790)$ & \\
\hline \multicolumn{8}{|l|}{ GSTM3 } \\
\hline High & 219 & $45(20.5 \%)$ & 3042 & Ref. & 0.804 & Ref. & 0.469 \\
\hline Low & 219 & $53(24.3 \%)$ & 2134 & $0.804(0.540-1.197)$ & & $0.860(0.571-1.295)$ & \\
\hline \multicolumn{8}{|l|}{ GSTM4 } \\
\hline High & 219 & $46(21.0 \%)$ & 3042 & Ref. & 0.387 & Ref. & 0.729 \\
\hline Low & 219 & $52(23.7 \%)$ & 2134 & $0.839(0.563-1.249)$ & & $0.930(0.618-1.400)$ & \\
\hline \multicolumn{8}{|l|}{ GSTM5 } \\
\hline High & 219 & $53(24.2 \%)$ & 2134 & Ref. & 0.495 & Ref. & 0.903 \\
\hline Low & 219 & 45 (20.5\%) & 2821 & $1.148(0.772-1.709)$ & & $0.975(0.647-1.468)$ & \\
\hline
\end{tabular}

Notes: *, adjustment for tumor stage

Abbreviations: GSTM, Glutathione S-transferase Mu; MST, median survival time; $\mathrm{HR}$, hazard ratio; $\mathrm{Cl}$, confidence interval 


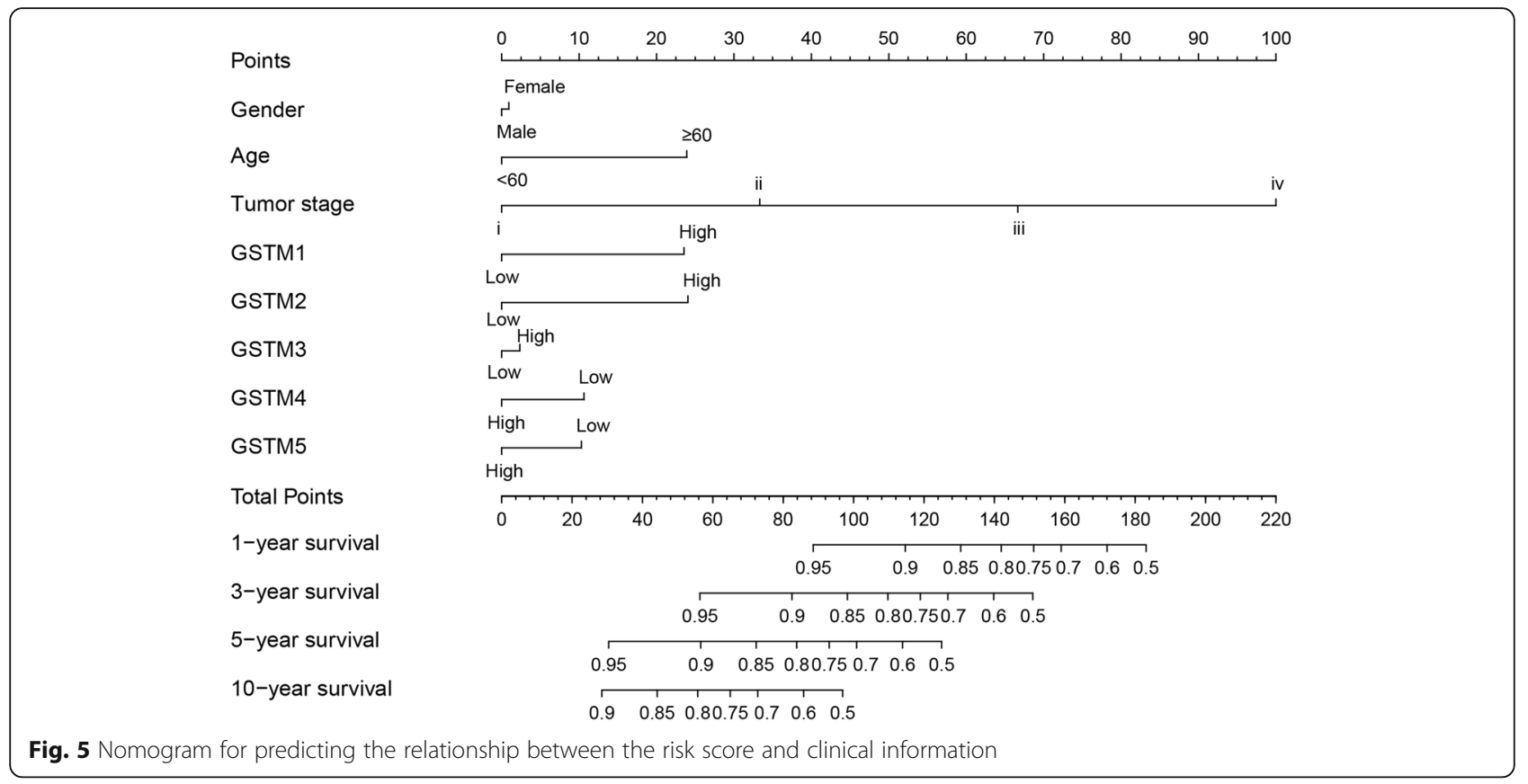

\section{Nomogram for predicting outcome}

The nomogram for predicting the prognostic value is shown in Fig. 5. Regarding clinical data, tumor stage provided the highest contribution risk score, and high expression of GSTM1 and GSTM2 showed higher contribution risk scores for COAD patients.

\section{Joint-effects survival analysis}

The grouping situation is summarized in Table 2. Group 1 showed the expression level combination related to favorable OS (low expression of GSTM1 and GSTM2). Group 3 included the combination associated with worse OS (high expression of GSTM1 and GSTM2). Compared with Group 3, Group 1 and Group 2 was related to favorable prognosis (all $P<0.05$, Fig. 6, Table 3 ).

\section{GSEA}

GSEA was performed to predict the effect of GSTM1 and GSTM2 low expression on prognosis. There were no statistically significant enrichment results for GSTM1 in both GO and KEGG analyses. The GO and KEGG

Table 2 The grouping information of joint-effects analysis

\begin{tabular}{ll}
\hline Group & Combinations \\
\hline 1 & Low GSTM1 + Low GSTM2 \\
2 & High GSTM1 + Low GSTM2 \\
& Low GSTM1 + High GSTM2 \\
3 & High GSTM1 + High GSTM2 \\
\hline
\end{tabular}

Abbreviations: GSTM, Glutathione S-transferase Mu enrichment results are shown in Fig. 7A-I and Fig. 8AI, respectively. For GO enrichment, low expression of GSTM2 was associated with cell division (Fig. 7B, D, E, $\mathrm{F}$, and I), cell cycle (Fig. 7A and $\mathrm{H}$ ), the NIF/NF- $\kappa B$ signaling pathway (Fig. 7G), and the ERAD pathway (Fig. 7C). For KEGG enrichment, low expression of GSTM2 was associated with cell metastasis (Fig. 8A), cell cycle (Fig. 8B, D, E, F, and G), activation of NF-kB (Fig. 8C), cell apoptosis (Fig. $8 \mathrm{H}$ ), and the WNT signaling pathway (Fig. 8I). The results are shown in Tables S2 and S3.

\section{Discussion}

In the current study, we investigated the expression level of the GSTM gene family in COAD, and performed a survival analysis including clinical data and GSTM gene expression. A nomogram model was used to predict the outcomes of COAD patients, and joint-effects survival analysis show that the combination of GSTM1 and GSTM2 low expression was a sensitive predictor of favorable prognosis. GSEA and serval enrichment analysis were performed to explain the effects of low expression level of GSTM1 and GSTM2 on prognosis.

GSTMs belong to the sub-family of soluble GSTs and include five members, GSTM1, GSTM2, GSTM3, GSTM4, and GSTM5 [8-11]. GSTs play important roles and are associated with glutathione (GSH) in the detoxification process [5-7]. Several GSTs are involved in the MAPK pathway, which controls cell proliferation, cell differentiation, and cell death, including the subfamilies GSTA, GSTP, and GSTM [21]. 


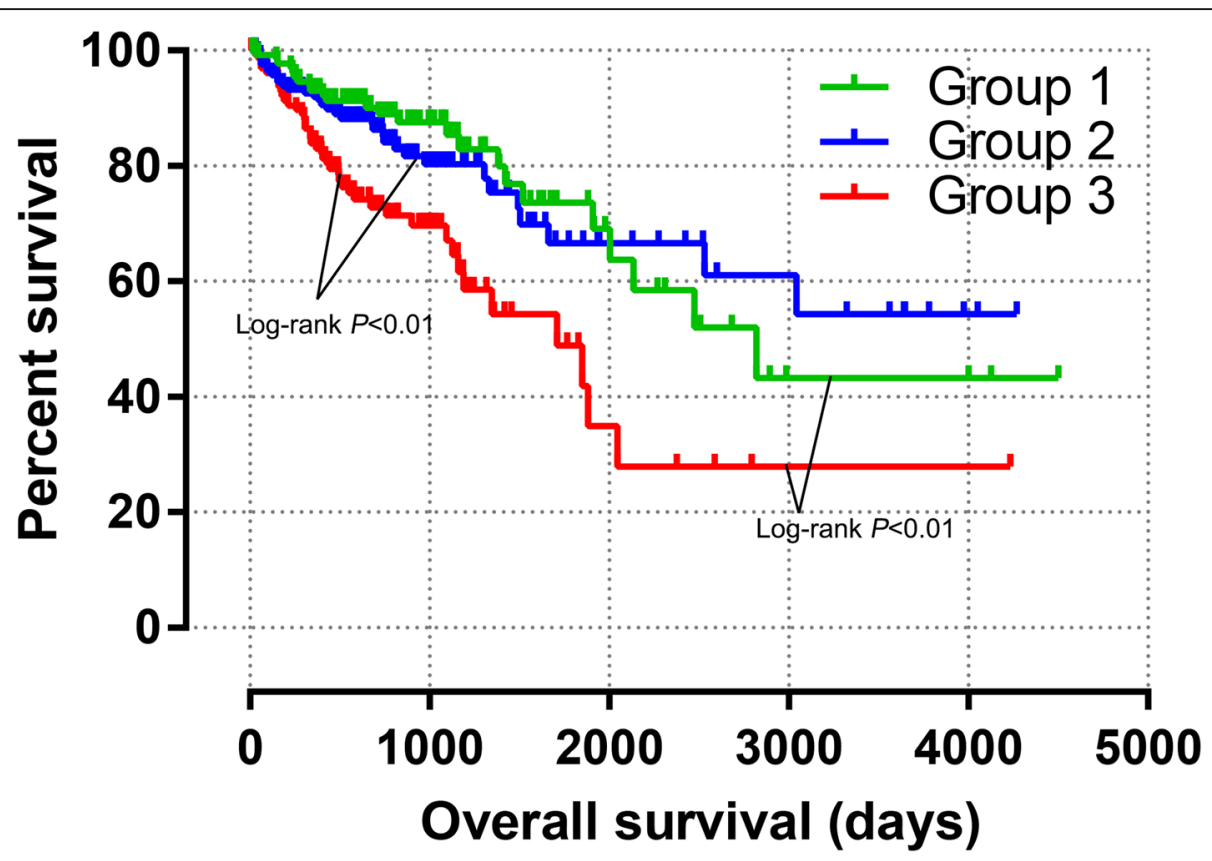

Fig. 6 Joint-effects analysis of GSTM1 and GSTM2 expression level combinations

GSTM1 is polymorphic in humans, and $40-60 \%$ of the population have a homozygous deletion of this gene [22]. Therefore, most studies of GSTM1 are performed using GSTM1-wt (wild-type genotype) and GSTM1-null (null genotype). Combining GSTM1 and p53 variants can divide colorectal cancer patients into several subgroups with significantly different prognosis, GSTM1+ polymorphism was associated with favorable OS in patients with colorectal cancer [23]. In ovarian cancer, GSTM1-null patients have a significant better survival than GSTM-wt patients, [24, 25] which could be attributed to the effect of GSTM1 on the expression of the p53 gene [25]. A previous study showed that GSTM1 induces tumor resistance by hydrolyzing tumor chemotherapy drugs or activating anti-apoptotic pathways, [26] and it was shown to be a negative regulator of apoptosis-related signaling cascades [22]. GSTM1 functions as a tumor suppressor gene in hepatocellular carcinoma; however, the prognostic value was not reported [27]. GSTM1 is also a risk factor of relapse in childhood acute lymphoblastic leukemia and hepatocellular carcinoma [24, 28, 29]. GSTM1 may also affect OS in breast cancer [30]. In gastric cancer, GSTM1-wt patients show better tumor-related and disease-free survival [31]. However, in the study of Acevedo et al., there was no significant correlation between GSTM1 polymorphisms and prognosis of prostate cancer [32].

GSTM2, a striated muscle-specific isozyme, [33] is highly expressed in mouse liver cancer, and involved in the Wnt/beta-catenin pathway [34]. In prostate cancer, GSTM2 is a potential tumor suppressor [35]. GSTM2 is among phase I or II metabolism-related genes, which were from phase II-conjugation [36]. These results are consistent with our GO enrichment results. GSTM2 is expressed at low levels in lung cancer [37]. There are no further reports about the relationship between the GSTM2 and cancer prognosis.

In the present study, low expression of GSTM1 and GSTM2 and their combination were associated with favorable OS in COAD patients. GSTM1 and GSTM2 are involved in cell cycle and detoxification, and tumorinhibiting cytokines may be degraded by the expression of GSTM1 and GSTM2 by speculating the results of enrichment analysis. However, GSTs can also degrade

Table 3 Joint-effects analysis of the prognostic value of combinations of GSTM1 and GSTM2

\begin{tabular}{llllllll}
\hline Group & $\begin{array}{l}\text { Patients } \\
(\mathrm{n}=438)\end{array}$ & No. of events (\%) & $\begin{array}{l}\text { MST } \\
(\text { days })\end{array}$ & $\begin{array}{l}\text { Crude } \\
P\end{array}$ & $\begin{array}{l}\text { Crude HR } \\
(95 \% \mathrm{Cl})\end{array}$ & $\begin{array}{l}\text { Adjusted } \\
P^{*}\end{array}$ & $\begin{array}{l}\text { Adjusted HR* } \\
(95 \% \mathrm{Cl})\end{array}$ \\
\hline $\mathbf{1}$ & 134 & $24(17.9 \%)$ & 2821 & $\mathbf{0 . 0 0 1}$ & $0.421(0.254-0.697)$ & $\mathbf{0 . 0 0 1}$ & $0.416(0.251-0.689)$ \\
$\mathbf{2}$ & 170 & $32(12.4 \%)$ & N/A & $\mathbf{0 . 0 0 1}$ & $0.467(0.293-0.744)$ & $\mathbf{0 . 0 0 1}$ & $0.469(0.294-0.689)$ \\
$\mathbf{3}$ & 134 & $42(31.3 \%)$ & 1711 & $<\mathbf{0 . 0 0 1}$ & Ref. & $<\mathbf{0 . 0 0 1}$ & Ref. \\
\hline
\end{tabular}

Notes: *, adjustment for tumor stage

Abbreviations: GSTM, Glutathione S-transferase Mu; MST, median survival time; HR, hazard ratio; $\mathrm{Cl}$, confidence interval 


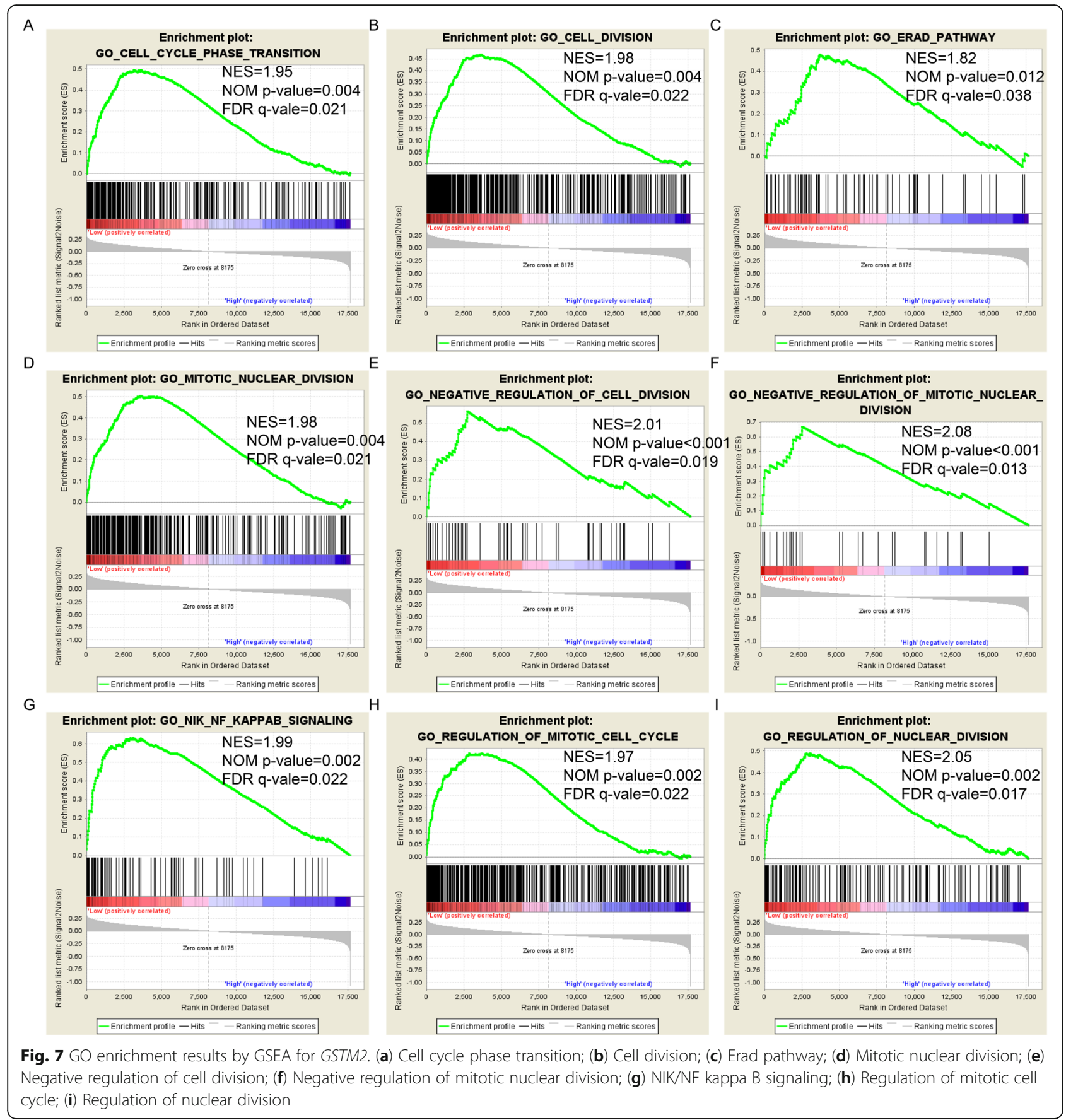

carcinogenic compounds. Therefore, further studies of the combination, connections, interactions, and synergy among GSTs family members are needed.

Regarding GSTM3, mutation of this gene may increase the risk of bladder cancer [38]. Polymorphisms of the GSTM4 gene are associated with increased risk of lung cancer [39] and could be used as a biomarker for the prediction of cisplatin response [40]. There are no reports on the relationship between cancer and the expression level of GSTM5.
The Wnt signaling pathway is critical for the development of colon cancer and patient outcome [41, 42]. GSTM2 is related to the Wnt signaling pathway, [34] which is consistent with the present enrichment results. This could explain the results showing that low expression of GSTM2 was related to favorable prognosis. In addition, predictive function of low GSTM2 and GSTM1 were involved in the cell cycle, which is associated with the occurrence of cancers and outcome. 


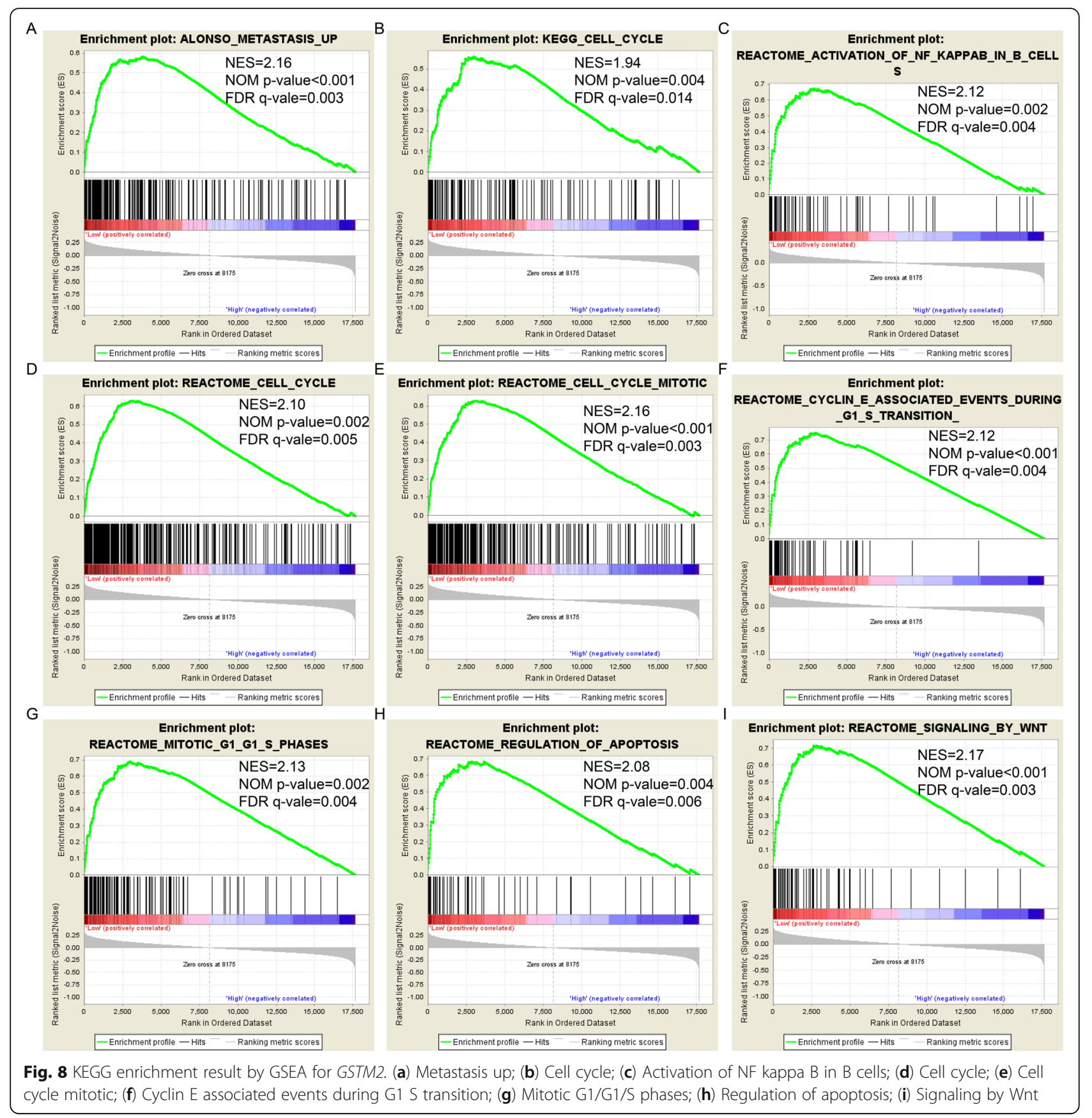

Previous studies of GSTM genes focused on the GSTM-null and GSTM-wt genotypes and their association with the risk and susceptibility to cancers. We found that low expression of GSTM1 and GSTM2 and their combination were correlated with favorable OS, and the nomogram showed that 1-, 5-, 10-year survival rates were affected by low expression levels of GSTM1 and GSTM2.

Our study had several disadvantages. First, further studies with a larger sample size are needed due to the small sample size of our study, and additional verification cohorts still need to verify our results. Second, due to the limited clinical data provided by TCGA, many factors affecting the prognosis of COAD cannot be included in the Cox model for correction. Third, because of the polymorphisms of GSTM genes, the genotype should also be included. Despite the above disadvantages, the present study is the first to report the relationship between the prognosis of COAD and GSTM gene family. These results suggest that low GSTM1 and GSTM2 expression was related to favorable prognosis in COAD. These two genes may be used as prognostic biomarkers for predicting the outcomes of COAD patients. 


\section{Conclusion}

Our study showed that the GSTM1 and GSTM2 expression was down-regulated in COAD, and low expression was markedly related to favorable prognosis. GSEA was performed to predict the function and mechanism. The results of GSEA indicated that the cell metabolism and detoxification functions of GSTM1 and GSTM2 may affect the prognosis of COAD patients. A nomogram including clinical information and gene expression levels was generated to predict the risk score for each factor. GSTM1 and GSTM2 seem interesting candidates for further studies aimed to validate their use as biomarkers of prognosis in COAD. Therefore, our findings can be used as preliminary support data for GSTM1 and GSTM2 as potential prognostic biomarkers for COAD. However, further studies are needed to confirm the present results.

\section{Supplementary information}

Supplementary information accompanies this paper at https://doi.org/10. 1186/s12881-020-01066-2. Additional file 1: Figure S1. GO functional enrichment analysis by
BiNGO of GSTM family.

Additional file 2: Figure S2. Gene interaction network for the GSTM1 gene and potentially related COAD gene cohort in TCGA.

Additional file 3: Figure S3. Gene interaction network for the GSTM2 gene and potentially related COAD gene cohort in TCGA.

Additional file 4: Table S1. Clinical information.

Additional file 5: Table S2. KEGG enrichment result by GSEA for GSTM2 (c2.all.v6.2.symbols.gmt).

Additional file 6: Table S3. GO enrichment results by GSEA for GSTM2 (c5.all.v6.2.symbols.gmt).

\section{Abbreviations}

COAD: Colon adenocarcinoma; GSTM: Glutathione S-transferase Mu; GSEA: Gene set enrichment analysis; TCGA: The Cancer Genome Atlas; GEPIA: Gene Expression Profiling Interactive Analysis; DAVID: Database for Annotation, Visualization, and Integrated Discovery; STRING: Search Tool for the Retrieval of Interacting Genes/Proteins; PPI: Protein-protein interaction; OS: Overall survival; MSigDB: Molecular Signatures Database; FDR: False discovery rate

\section{Acknowledgements}

The authors thank the contributors of TCGA (https://cancergenome.nih.gov/) for sharing their data on open access.

\section{Authors' contributions}

EG and XZ designed and wrote this manuscript. EG, HW, XL, LW, and XZ conducted and further performed the study, processed and analyzed the data. XZ has rigorously revised the final draft. All authors read and approved the manuscript and agree to be accountable for all aspects of the research in ensuring that the accuracy or integrity of any part of the work are appropriately investigated and resolved.

\section{Funding}

The present study was supported in part by the National Nature Science Foundation of China (grant no. 81360448), International Communication of Guangxi Medical University Graduate Education (2017), the Self-Raised Scientific Research Fund of the Health and Family Planning Commission of the Guangxi Zhuang Autonomous Region (grant no. Z2015198) and the Nanning Scientific Research and Technology Development Project (Key Research and
Development Plan); grant no. 20173018-3). The funders had no role in study design, data collection and analysis, decision to publish, or preparation of the manuscript.

\section{Availability of data and materials}

The raw datasets used during the present study can be downloaded from The Cancer Genome Atlas (https://portal.gdc.cancer.gov/projects/TCGACOAD). The COAD RNA-seq dataset are open assess to everyone and can be downloaded directly from The Cancer Genome Atlas website without any login account.

\section{Ethics approval and consent to participate}

Since all datasets of COAD included in the present study were downloaded from The Cancer Genome Atlas (https://portal.gdc.cancer.gov/projects/TCGA(OAD), and the data acquisition is open access to everyone. Therefore, additional approval by an Ethics Committee was not needed.

\section{Consent for publication}

Not applicable.

\section{Competing interests}

The authors declare that they have no competing interests.

\section{Author details}

${ }^{1}$ School of Public Health, Guangxi Medical University, 22 Shuang Yong Road, Nanning 530021, Guangxi Zhuang Autonomous Region, People's Republic of China. ${ }^{2}$ School of International Education, Guangxi Medical University, Nanning 530021, Guangxi Zhuang Autonomous Region, People's Republic of China. ${ }^{3}$ Department of gastrointestinal surgery, The Third Affiliated Hospital of Guangxi Medical University, Nanning 530031, Guangxi Zhuang Autonomous Region, People's Republic of China. ${ }^{4}$ Department of Hepatobiliary Surgery, The First Affiliated Hospital of Guangxi Medical University, Nanning 530021, Guangxi Zhuang Autonomous Region, People's Republic of China.

Received: 21 March 2020 Accepted: 8 June 2020

Published online: 15 June 2020

\section{References}

1. Siegel RL, Miller KD, Jemal A. Cancer statistics, 2018. CA Cancer J Clin. 2018; 68(1):7-30.

2. Hrasovec S, Glavac D. MicroRNAs as novel biomarkers in colorectal Cancer. Front Genet. 2012;3:180.

3. Watson AJ, Collins PD. Colon cancer: a civilization disorder. Dig Dis. 2011; 29(2):222-8.

4. Cunningham D, Atkin W, Lenz HJ, Lynch HT, Minsky B, Nordlinger B, et al. Colorectal cancer. Lancet. 2010;375(9719):1030-47.

5. Dasari S, Ganjayi MS, Yellanurkonda P, Basha S, Meriga B. Role of glutathione S-transferases in detoxification of a polycyclic aromatic hydrocarbon, methylcholanthrene. Chem Biol Interact. 2018;294:81-90.

6. Hayes JD, Flanagan JU, Jowsey IR. Glutathione transferases. Annu Rev Pharmacol Toxicol. 2005;45:51-88.

7. Oakley A. Glutathione transferases: a structural perspective. Drug Metab Rev. 2011;43(2):138-51.

8. Pearson WR, Vorachek WR, Xu SJ, Berger R, Hart I, Vannais D, et al. Identification of class-mu glutathione transferase genes GSTM1-GSTM5 on human chromosome 1p13. Am J Hum Genet. 1993;53(1):220-33.

9. DeJong $\mathrm{JL}$, Mohandas T, Tu CP. The human Hb (mu) class glutathione Stransferases are encoded by a dispersed gene family. Biochem Biophys Res Commun. 1991;180(1):15-22.

10. Mannervik B, Awasthi YC, Board PG, Hayes JD, Di llio C, Ketterer B, et al. Nomenclature for human glutathione transferases. Biochem J. 1992;282(Pt 1):305-6.

11. Mannervik B, Board PG, Hayes JD, Listowsky I, Pearson WR. Nomenclature for mammalian soluble glutathione transferases. Methods Enzymol. 2005; 401:1-8.

12. Tang Z, Li C, Kang B, Gao G, Li C, Zhang Z. GEPIA: a web server for cancer and normal gene expression profiling and interactive analyses. Nucleic Acids Res. 2017;45(W1):W98-W102. 
13. Huang da W, Sherman BT, Lempicki RA. Bioinformatics enrichment tools: paths toward the comprehensive functional analysis of large gene lists. Nucleic Acids Res 2009;37(1):1-13.

14. Huang da W, Sherman BT, Lempicki RA. Systematic and integrative analysis of large gene lists using DAVID bioinformatics resources. Nat Protoc 2009; 4(1):44-57.

15. Maere S, Heymans K, Kuiper M. BiNGO: a Cytoscape plugin to assess overrepresentation of gene ontology categories in biological networks. Bioinformatics. 2005;21(16):3448-9.

16. Warde-Farley D, Donaldson SL, Comes O, Zuberi K, Badrawi R, Chao P, et al. The GeneMANIA prediction server: biological network integration for gene prioritization and predicting gene function. Nucleic acids research. 2010; 38(Web Server issue):W214-20.

17. Szklarczyk D, Franceschini A, Wyder S, Forslund K, Heller D, Huerta-Cepas J, et al. STRING v10: protein-protein interaction networks, integrated over the tree of life. Nucleic Acids Res. 2015:43(Database issue):D447-52.

18. Balachandran VP, Gonen M, Smith JJ, Dematteo RP. Nomograms in oncology - more than meets the eye. Lancet Oncol. 2015;16(4):e173.

19. Subramanian A, Tamayo P, Mootha VK, Mukherjee S, Ebert BL, Gillette MA, et al. Gene set enrichment analysis: a knowledge-based approach for interpreting genome-wide expression profiles. Proc Natl Acad Sci U S A. 2005;102(43):15545-50.

20. Shannon P, Markiel A, Ozier O, Baliga NS, Wang JT, Ramage D, et al. Cytoscape: a software environment for integrated models of biomolecular interaction networks. Genome Res. 2003;13(11):2498-504.

21. Laborde E. Glutathione transferases as mediators of signaling pathways involved in cell proliferation and cell death. Cell Death Differ. 2010;17(9): 1373-80.

22. Hosono N, Kishi S, Iho S, Urasaki Y, Yoshida A, Kurooka H, et al. Glutathione S-transferase M1 inhibits dexamethasone-induced apoptosis in association with the suppression of Bim through dual mechanisms in a lymphoblastic leukemia cell line. Cancer Sci. 2010;101(3):767-73.

23. Csejtei A, Tibold A, Varga Z, Koltai K, Ember A, Orsos Z, et al. GSTM, GSTT and p53 polymorphisms as modifiers of clinical outcome in colorectal cancer. Anticancer Res. 2008;28(3B):1917-22.

24. Medeiros R, Pereira D, Afonso N, Palmeira C, Faleiro C, Afonso-Lopes C, et al. Platinum/paclitaxel-based chemotherapy in advanced ovarian carcinoma: glutathione S-transferase genetic polymorphisms as predictive biomarkers of disease outcome. Int J Clin Oncol. 2003;8(3):156-61.

25. Howells RE, Holland T, Dhar KK, Redman CW, Hand P, Hoban PR, et al. Glutathione S-transferase GSTM1 and GSTT1 genotypes in ovarian cancer: association with p53 expression and survival. Int J Gynecol Cancer. 2001; 11(2):107-12.

26. Fu XT, Song K, Zhou J, Shi YH, Liu WR, Tian MX, et al. Autophagy activation contributes to glutathione transferase mu 1-mediated chemoresistance in hepatocellular carcinoma. Oncol Lett. 2018;16(1):346-52.

27. Song K, Yi J, Shen X, Cai Y. Genetic polymorphisms of glutathione Stransferase genes GSTM1, GSTT1 and risk of hepatocellular carcinoma. PLoS One. 2012;7(11):e48924.

28. Hall AG, Autzen P, Cattan AR, Malcolm AJ, Cole M, Kernahan J, et al. Expression of mu class glutathione S-transferase correlates with event-free survival in childhood acute lymphoblastic leukemia. Cancer Res. 1994;54(20): $5251-4$.

29. Shen YH, Chen S, Peng YF, Shi YH, Huang XW, Yang GH, et al. Quantitative assessment of the effect of glutathione S-transferase genes GSTM1 and GSTT1 on hepatocellular carcinoma risk. Tumour Biol. 2014;35(5):4007-15.

30. Wang $X$, Huang ZH. Predictive potential role of glutathione S-transferase polymorphisms in the prognosis of breast cancer. Genet Mol Res. 2015; 14(3):10236-41.

31. Ott K, Lordick F, Becker K, Ulm K, Siewert J, Hofler H, et al. Glutathione-Stransferase P1, T1 and M1 genetic polymorphisms in neoadjuvant-treated locally advanced gastric cancer: GSTM1-present genotype is associated with better prognosis in completely resected patients. Int J Color Dis. 2008;23(8): 773-82.

32. Acevedo CA, Quinones LA, Catalan J, Caceres DD, Fulla JA, Roco AM. Impact of CYP1A1, GSTM1, and GSTT1 polymorphisms in overall and specific prostate cancer survival. Urol Oncol. 2014;32(3):280-90.

33. Anttila S, Hirvonen A, Vainio H, Husgafvel-Pursiainen K, Hayes JD, Ketterer B. Immunohistochemical localization of glutathione S-transferases in human lung. Cancer Res. 1993;53(23):5643-8.
34. Giera S, Braeuning A, Kohle C, Bursch W, Metzger U, Buchmann A, et al. Wnt/beta-catenin signaling activates and determines hepatic zonal expression of glutathione S-transferases in mouse liver. Toxicol Sci. 2010; 115(1):22-33.

35. Davalieva K, Kostovska IM, Kiprijanovska S, Markoska K, Kubelka-Sabit K, Filipovski $\mathrm{V}$, et al. Proteomics analysis of malignant and benign prostate tissue by 2D DIGE/MS reveals new insights into proteins involved in prostate cancer. Prostate. 2015;75(14):1586-600.

36. Nishimura J, Dewa Y, Okamura T, Muguruma M, Jin M, Saegusa Y, et al. Possible involvement of oxidative stress in fenofibrate-induced hepatocarcinogenesis in rats. Arch Toxicol. 2008;82(9):641-54.

37. Tang SC, Wu MF, Wong RH, Liu YF, Tang LC, Lai CH, et al. Epigenetic mechanisms for silencing glutathione S-transferase $\mathrm{m} 2$ expression by hypermethylated specificity protein 1 binding in lung cancer. Cancer. 2011; 117(14):3209-21.

38. Schnakenberg E, Breuer R, Werdin R, Dreikorn K, Schloot W. Susceptibility genes: GSTM1 and GSTM3 as genetic risk factors in bladder cancer. Cytogenet Cell Genet. 2000;91(1-4):234-8.

39. Liloglou T, Walters M, Maloney P, Youngson J, Field JK. A T2517C polymorphism in the GSTM4 gene is associated with risk of developing lung cancer. Lung Cancer. 2002;37(2):143-6.

40. Moyer AM, Sun Z, Batzler AJ, Li L, Schaid DJ, Yang P, et al. Glutathione pathway genetic polymorphisms and lung cancer survival after platinumbased chemotherapy. Cancer Epidemiol Biomark Prev. 2010;19(3):811-21.

41. Ionov Y, Peinado MA, Malkhosyan S, Shibata D, Perucho M. Ubiquitous somatic mutations in simple repeated sequences reveal a new mechanism for colonic carcinogenesis. Nature. 1993;363(6429):558-61.

42. Abdul Khalek FJ, Gallicano Gl, Mishra L. Colon cancer stem cells. Gastrointest Cancer Res. 2010(Suppl 1):S16-23.

\section{Publisher's Note}

Springer Nature remains neutral with regard to jurisdictional claims in published maps and institutional affiliations.
Ready to submit your research? Choose BMC and benefit from:

- fast, convenient online submission

- thorough peer review by experienced researchers in your field

- rapid publication on acceptance

- support for research data, including large and complex data types

- gold Open Access which fosters wider collaboration and increased citations

- maximum visibility for your research: over $100 \mathrm{M}$ website views per year

At BMC, research is always in progress.

Learn more biomedcentral.com/submissions 